Y. Teranishi

Nagoya Math. J.

Vol. 99 (1985), 131-146

\title{
THE FUNCTIONAL EQUATION OF ZETA DISTRIBUTIONS ASSOCIATED WITH PREHOMOGENEOUS VECTOR SPACES $(\tilde{G}, \tilde{\rho}, M(n, C))$
}

\author{
YASUO TERANISHI
}

\section{Introduction}

Let $(G, \rho, V)$ be a triple of a linear algebraic group $G$ and a rational representation $\rho$ on a finite dimensional vector space $V$, all defined over the complex number field $C$.

We call the triple $(G, \rho, V)$ a prehomogeneous vector space if $G$ has a Zariski-open orbit. Assume that the triple $(G, \rho, V)$ is a prehomogeneous vector space. Then there exists a proper algebraic subset $S$ of $V$ such that $V-S$ is a single $G$-orbit. The algebraic set $S$ is called the singular set of $(G, \rho, V)$. For a rational character of $G$, a non-zero rational function $P$ on $V$ is called a relative invariant of $(G, \rho, V)$ corresponding to $\chi$ if

$$
P(\rho(g) x)=\chi(g) P(x) \quad(g \in G, x \in V) .
$$

Let $P_{1}, \cdots, P_{n}$ be irreducible polynomials defining the components of $S$ with codimension 1. It is known that $P_{1}, \cdots, P_{n}$ are relative invariants of $(G, \rho, V)$ (cf. [1]). The set $\left\{P_{1}, \cdots, P_{n}\right\}$ is called a complete set of irreducible relative invariants of $(G, \rho, V)$.

The purpose of this paper is to give an explicit expression for the Fourier transform of relative invariants on a certain class of prehomogeneous vector spaces.

Notation. We denote by $\boldsymbol{Z}, \boldsymbol{R}$ and $\boldsymbol{C}$ the ring of integers, the rational number field and the complex number field, respectively. For $z \in C$, we set $e(z)=\exp (2 \pi \sqrt{-1} z)$. We denote by $M(n, C)$ (resp. $M(n, R))$ the complex (resp. real) vector space consisting of all $n$ by $n$ matrices with entries in $C$ (resp. $R$ ). For any matrix $x,{ }^{t} x$ denotes the transposed matrix. For

Received June 9, 1984. 
$x \in M(n, C)$, we set $x^{*}={ }^{t} x^{-1}$. For a $C^{\infty}$-manifold $X, C_{0}^{\infty}(X)$ denotes the space of $C^{\infty}$-functions with compact support on $X$. We denote by $\Gamma(z)$ the usual Gamma function. We denote by $B_{n}(C)$ (resp. $B_{n}(R)$ ) the subgroup of the general linear group $G L(n, C)$ (resp. $G L(n, R)$ ) consisting of all upper triangular matrices.

\section{$\S 1$. Prehomogeneous vector space $(\tilde{G}, \tilde{\rho}, M(n, C))$}

1.1. Let $G$ be a linear algebraic group, $\rho: G \rightarrow G L(n, C)$ a rational representation of $G$ both defined over $C$. We denote by $\tilde{G}$ the direct product group $G \times B_{n}(C)$. For any $x \in M(n, C)$ and $\tilde{g}=(g, a) \in \tilde{G}$, set $\tilde{\rho}(\tilde{g}) x=\rho(g) x a^{-1}$. Then $\tilde{\rho}$ is a rational representation of $\tilde{G}$. We denote by $\tilde{\rho}^{*}$ the contragredient representation to $\tilde{\rho}$. It is known that the triple $(\tilde{G}, \tilde{\rho}, M(n, C))$ is a prehomogeneous vector space if and only if the triple $\left(G, \tilde{\rho}^{*}, M(n, C)\right)$ is a prehomogeneous vector space. In what follows we assume that the triplet $(\tilde{G}, \tilde{\rho}, M(n, C))$ is a P.V. Let $\left\{P_{0}, \cdots, P_{k}\right\}$ be a complete set of irreducible relative invariants of $(\tilde{G}, \tilde{\rho}, M(n, C))$ and $\chi_{0}, \cdots, \chi_{k}$ characters of $P_{0}, \cdots, P_{k}$, respectively. Since $\operatorname{det} x$ is an irreducible relative invariant of $(\tilde{G}, \tilde{\rho}, M(n, C))$, we may set $P_{0}(x)=\operatorname{det} x$. Let $P(x)$ be any relative invariant polynomial of $(\tilde{G}, \tilde{\rho}, M(n, C))$. For any $x \in M(n, C))$, we denote by $x^{\ell}$ the $\ell$-th column vector of $x$. Then it is known that $P(x)$ is homogeneous with respect to each column vector $x^{\ell}(1 \leq \ell \leq n)$. Denoting by $\lambda_{\ell}$ the homogeneous degree of $P(x)$ with respect to $x^{\ell}$, one can show that $\lambda_{\ell}$ 's satisfy

$$
\lambda_{1} \geq \lambda_{2} \geq \cdots \geq \lambda_{n}, \quad \text { (cf. [6]) }
$$

Denoting by $\lambda$ the $n$-tuple $\left(\lambda_{1}, \cdots, \lambda_{n}\right)$, we call $\lambda$ the partition corresponding to the relative invariant polynomial $P(x)$. Let $\lambda(0), \cdots, \lambda(k)$ be partitions corresponding to $P_{0}, \cdots, P_{k}$, respectively. We set

$$
\begin{aligned}
& P_{0}^{*}(x)=P_{0}(x)=\operatorname{det} x, \quad \chi_{0}^{*}=\chi_{0}, \\
& P_{i}^{*}(x)=P_{i}\left(x^{*}\right) P_{0}(x)^{\lambda(i)_{1}} \quad \text { and } \quad \chi_{i}^{*}=\chi_{i}^{-1} \chi_{0}^{\lambda(i)_{1}} \quad(1 \leq i \leq k) .
\end{aligned}
$$

Then one sees easily that $P_{0}^{*}, \cdots, P_{k}^{*}$ are relative invariants of $\left(\tilde{G}, \tilde{\rho}^{*}\right.$, $M(n, C))$ and satisfy

$$
P_{i}^{*}\left(\tilde{\rho}^{*}(\tilde{g}) x\right)=\chi_{i}^{*}(\tilde{g})^{-1} P_{i}(x) \quad(0 \leq i \leq k) .
$$

Moreover the set $\left\{P_{0}^{*}, \cdots, P_{k}^{*}\right\}$ is a complete set of irreducible relative invariants of $\left(\tilde{G}, \tilde{\rho}^{*}, M(n, C)\right)$ (cf. [6]). 
We denote by $X_{\rho}(\tilde{G})$ the group of all rational characters corresponding to the relative invariants of $(\tilde{G}, \tilde{\rho}, M(n, C))$. It is known that the group $X_{\rho}(\tilde{G})$ is a free abelian group of rank $k+1$ generated by $\chi_{0}, \cdots, \chi_{k}$ and hence there exists $k+1$-tuples $\left(\delta\left(\chi_{0}\right), \cdots, \delta\left(\chi_{k}\right)\right)$ and $\left(\delta^{*}\left(\chi_{0}\right), \cdots, \delta^{*}\left(\chi_{k}\right) \in Z^{k+1}\right.$ such that

$$
\chi=\prod_{i=0}^{k} \chi_{i}^{\delta(x) i}=\prod_{i=0}^{k} \chi_{i}^{* \delta *(x) i}, \quad \text { (cf. [6]). }
$$

For any $\chi \in X_{\rho}(\tilde{G})$, we set

$$
\begin{gathered}
P_{\chi}=\prod_{i=0}^{k} P_{i}^{\delta(\chi) i}, \quad P_{\chi}^{*}=\prod_{i=0}^{k} P_{i}^{* \delta *(\chi) i}, \\
\delta(\chi)=\left(\delta(\chi)_{0}, \cdots, \delta(\chi)_{k}\right) \quad \text { and } \quad \delta^{*}(\chi)=\left(\delta(\chi)_{0}, \cdots, \delta^{*}(\chi)_{k}\right) .
\end{gathered}
$$

Let $\lambda(0), \cdots, \lambda(k)$ be partitions of $P_{0}, \cdots, P_{k}$, respectively. For any $s=\left(s_{0}, \cdots, s_{k}\right) \in C^{k+1}$, we write

$$
\lambda_{\ell}(s)=\sum_{i=0}^{k} s_{i} \lambda(i)_{\ell}
$$

and

$$
\lambda_{\ell}^{*}(s)=s_{0}+\sum_{i=1}^{k} s_{i}\left(\lambda(i)_{1}-\lambda(i)_{\ell}\right) \quad(1 \leq \ell \leq n) .
$$

Furthermore we set

$$
\gamma(s)=\prod_{\ell=1}^{n} \Gamma\left(\lambda_{\ell}(s)+n-\ell+1\right)
$$

and

$$
\gamma^{*}(s)=\prod_{\ell=1}^{n} \Gamma\left(\lambda_{\ell}^{*}(s)+n-\ell+1\right) .
$$

Then the " $b$-function" of $(\tilde{G}, \tilde{\rho}, M(n, C))(\operatorname{resp} .(\tilde{G}, \tilde{\rho}, M(n, C))$ is given by

$$
b_{x}(s)=\frac{\gamma(s)}{\gamma(s-\delta(\chi))} \quad\left(\text { resp. } b_{x}^{*}(s)=\frac{\delta^{*}(s)}{\gamma^{*}\left(s-\delta^{*}(\chi)\right)}\right), \quad(\text { cf. [6]). }
$$

For $s=\left(s_{0}, s_{1}, \cdots, s_{k}\right) \in C^{k+1}$, we set

$$
s^{*}=\left(s_{0}^{*}, s_{1}^{*}, \cdots, s_{k}^{*},\right.
$$

where $s_{0}^{*}=-\lambda_{1}(s)$ and $s_{i}^{*}=s_{i}(1 \leq i \leq k)$.

Lemma 1. Notations being as above, one hi...

(i ) $\lambda_{\ell}^{*}\left(s^{*}\right)=-\lambda_{\ell}(s)(1 \leq \ell \leq n)$,

(ii) $\delta(\chi)^{*}=-\delta^{*}(\chi)$. 
Proof. ' ( i ) $\lambda_{\ell}^{*}\left(s^{*}\right)=\sum_{i=1}^{k} s_{i}\left(\lambda(i)_{1}-\lambda(i)_{\ell}\right)-\sum_{i=0}^{k} s_{i} \lambda(i)_{1}$

$$
\begin{aligned}
& =-\sum_{i=0}^{k} s_{i} \lambda(i)_{\ell} \\
& =-\lambda_{\ell}(s)
\end{aligned}
$$

(ii) $\delta(\chi)^{*}=\left(-\lambda_{1}(\delta(\chi)), \delta(\chi)_{1}, \cdots, \delta(\chi)_{k}\right)$

$$
=\left(-\sum_{i=0}^{k} \delta(\chi)_{i} \lambda(i)_{1}, \delta(\chi)_{1} \cdots, \delta(\chi)_{k}\right) .
$$

Then one sees immediately

$$
\delta^{*}(\chi)=\left(\sum_{i=0}^{k} \delta(\chi)_{i} \lambda(i)_{1},-\delta(\chi)_{1}, \cdots, \delta(\chi)_{k}\right) .
$$

If $x$ is not contained in the singular set of $(\tilde{G}, \tilde{\rho}, M(n, C))$, it follows from the definition of $P_{x}^{*}(x)$ that

$$
P_{x}^{*}\left(x^{*}\right)=P_{x}(x)^{-1}
$$

For $\chi \in X_{\rho}(\tilde{G})$, we set

$$
d(\chi)=\sum_{i=0}^{k} \delta(\chi)_{i} \operatorname{deg} P_{i} \quad \text { and } \quad d^{*}(\chi)=\sum_{i=0}^{k} \delta^{*}(\chi)_{i} \operatorname{deg} P_{i}^{*} .
$$

1.2. In the following, we assume that $G$ is defined over $\boldsymbol{R}$. Denoting by $G_{R}$ the set of $R$-rational points of $G$, we set

$$
\begin{aligned}
\tilde{G}_{R} & =G \times B_{n}(R), \\
S_{R} & =S \cap M(n, R), \\
S_{R}^{*} & =S^{*} \cap M(n, R), \\
\tilde{\rho}_{R} & =\tilde{\rho}_{\tilde{\sigma}_{R}} .
\end{aligned}
$$

Furthermore we always assume the following conditions:

(A.1) $G_{R}$ is a connected subgroup of $G L(n, R)$.

(A.2) the singular set $S$ of $(\tilde{G}, \tilde{\rho}, M(n, C))$ is the union of irreducible hypersurfaces of the form

$$
S_{i}=\left\{x \in M(n, R) ; P_{i}(x)=0\right\} \quad(0 \leq i \leq k),
$$

where, for each $i, P_{i}(x)$ is a $C$-irreducible polynomial with real coefficients.

(A.3) $\quad M(n, R)-S_{R}$ is a single $\tilde{\rho}_{R}\left(\tilde{G}_{R}\right)$-orbit.

We denote by $\tilde{G}_{R}^{0}$ the connected components of the identity and conicer the $\tilde{\rho}_{R}\left(\tilde{G}_{R}^{0}\right)$-orbital decomposition of $M(n, R)-S_{R}$

$$
M(n, R)-S_{R}=V_{1} \cup \cdots \cup V_{\nu} .
$$


For $\tilde{\rho}_{R}\left(G_{R}^{0}\right)$-orbit $V_{i}$, we set

$$
V_{i}^{*}=\left\{x \in M(n, R) ; x^{*} \in V_{i}\right\} .
$$

Then one sees that the set $M(n, R)-S_{R}^{*}$ is decomposed into the disjoint union of $\tilde{\rho}_{R}^{*}\left(G_{R}^{0}\right)$-orbits

$$
M(n, R)-S_{R}^{*}=V_{1}^{*} \cup \cdots \cup V_{\nu}^{*} .
$$

For $s=\left(s_{0}, \cdots, s_{k}\right) \in C^{h+1}$, we set

$$
\begin{aligned}
|P(x)|^{s} & =\prod_{i=0}^{k}\left|P_{i}(x)\right|^{s_{i}}, & \left|P^{*}(x)\right|^{s} & =\prod_{i=0}^{k}\left|P_{1}^{*}(x)\right|^{s_{i}}, \\
|\chi(g)|^{s} & =\prod_{i=0}^{k}\left|\chi_{i}(g)\right|^{s_{i}}, & \left|\chi^{*}(g)\right|^{s} & =\prod_{i=0}^{k}\left|\chi_{i}^{*}(g)\right|^{s_{i}}
\end{aligned}
$$

\section{§2. Fourier transforms of relative invariants}

2.1. We denote by $S(M(n, R))$ the Schwartz space of the vector space $M(n, R)$. We consider the following integrals:

$$
\Phi_{i}(f, s)=\int_{V_{i}} f(x)|P(x)|^{s} d x
$$

and

$$
\Phi_{i}^{*}(f, s)=\int_{V_{i}^{*}} f(x)\left|P^{*}(x)\right|^{s} d x \quad(1 \leq i \leq \nu)
$$

where $d x$ is the Euclidean measure on $V_{i}$. If $\operatorname{Re}(s)_{0}>0, \cdots, \operatorname{Re}(s)_{k}>0$, the above integrals $\Phi_{i}(f, s), \Phi_{i}^{*}(f, s)$ are absolutely convergent.

For $\chi \in X_{o}(\tilde{G})$, we set

$$
\varepsilon_{i}(\chi)=\left.\operatorname{sgn} P_{\chi}\right|_{V_{i}} \text { and } \varepsilon_{i}^{*}(\chi)=\left.\operatorname{sgn} P_{\chi}^{*}\right|_{V_{i}^{*}} \quad(1 \leq i \leq \nu) .
$$

By $(1)$, one has $\varepsilon_{i}(\chi)=\varepsilon_{i}^{*}(\chi),(1 \leq i \leq \nu)$. We also set, for $s=\left(s_{0}, \cdots, s_{k}\right)$ $\in C^{k+1}$,

$$
\begin{aligned}
& d(s)=\sum_{\ell=0}^{k} s_{\ell} \operatorname{deg} P_{\ell}, \quad d^{*}(s)=\sum_{\ell=0}^{k} s_{\ell} \operatorname{deg} P_{\ell}^{*} \\
& \varepsilon_{i}(s)=e\left(\frac{1}{4} \sum_{\ell=0}^{k} s_{\ell}\left(1-\varepsilon_{i}\left(\chi_{\ell}\right)\right)\right), \quad \varepsilon_{i}^{*}(s)=e\left(\frac{1}{4} \sum s_{\ell}\left(1-\varepsilon_{i}^{*}\left(\chi_{\ell}^{*}\right)\right)\right) .
\end{aligned}
$$

Then, one can easily check:

$$
\begin{aligned}
& d(\chi)=d(\delta(\chi)), \quad d^{*}(\chi)=d^{*}\left(\delta^{*}(\chi)\right), \\
& \varepsilon_{i}(\chi)=\varepsilon_{i}(\delta(\chi)), \quad \varepsilon_{i}^{*}(\chi)=\varepsilon_{i}^{*}\left(\delta^{*}(\chi)\right), \\
& d(s)=-d^{*}\left(s^{*}\right) \quad \text { and } \quad d(\chi)=d^{*}(\chi) .
\end{aligned}
$$


We set

$$
F_{i}(f, s)=\frac{1}{\gamma(s)} \Phi_{i}(f, s) \quad \text { and } \quad F_{i}^{*}(f, s)=\frac{1}{\gamma^{*}(s)} \Phi_{i}^{*}(f, s) .
$$

Denoting by $\hat{f}$ the Fourier transform of $f$, one can easily prove the following

LEMMA 2. If $\operatorname{Re}\left(s_{0}\right), \cdots, \operatorname{Re}\left(s_{k}\right)$ are sufficiently large, one has

(i ) for any $\chi \in X_{\rho}(\tilde{G})$, such that $\delta^{*}(\chi)_{0}, \cdots, \delta^{*}(\chi)_{k} \geq 0$,

$$
F_{i}\left(p_{\chi}^{*} f, s\right)=(-2 \pi \sqrt{-1})^{-d^{*}(\chi)} \varepsilon_{i}(\chi) F_{i}(\hat{f}, s-\delta(\chi))
$$

and for any $\chi \in X_{\rho}(\tilde{G})$ such that $\delta(\chi)_{0}, \cdots, \delta(\chi)_{k} \geq 0$,

$$
F_{i}^{*}\left(\widehat{P_{\chi} \cdot f}, s\right)=(2 \pi \sqrt{-1})^{-d(\chi)} \varepsilon_{i}(\chi) F_{i}^{*}\left(\hat{f}, s-\delta^{*}(\chi)\right)
$$

(ii) for any $\chi \in X_{\rho}(\tilde{G})$ such that $\delta(\chi)_{0}, \cdots, \delta(\chi)_{k} \geq 0$,

$$
F_{i}\left(\widehat{P_{\chi}(\mathrm{grad}) \cdot f}, s\right)=(-2 \pi \sqrt{-1})^{d(\chi)} \varepsilon_{i}(\chi) b_{\chi}(s+\delta(\chi)) F_{i}(\hat{f}, s+\delta(\chi))
$$

and for any $\chi \in X_{\rho}(\tilde{G})$ such that $\delta^{*}(\chi)_{0}, \cdots, \delta^{*}(\chi)_{k} \geq 0$,

$$
F_{i}^{*}\left(\widehat{P_{\chi}^{*}(\operatorname{grad})} f, s\right)=(2 \pi \sqrt{-1})^{d^{*}(\chi)} \varepsilon_{i}^{*}(\chi) b_{\chi}^{*}\left(s+\delta^{*}(\chi)\right) F_{i}^{*}\left(\hat{f}, s+\delta^{*}(\chi)\right) .
$$

(iii) for any $\chi \in X_{\rho}(\tilde{G})$ such that $\delta^{*}(\chi)_{0}, \cdots, \delta^{*}(\chi)_{k} \geq 0$,

$$
F_{i}\left(P_{\chi}^{*}(\operatorname{grad}) f, s\right)=\varepsilon_{i}(\chi)(-1)^{d^{*}(x)} F_{i}(f, s-\delta(\chi))
$$

and for any $\chi \in X_{\rho}(\tilde{G})$ such that $\delta(\chi)_{0}, \cdots, \delta(\chi)_{k} \geq 0$,

$$
F_{i}^{*}\left(P_{\chi}(\operatorname{grad}) f, s\right)=\varepsilon_{i}^{*}(\chi)(-1)^{d(\chi)} F_{i}^{*}\left(f, s-\delta^{*}(\chi)\right) .
$$

Let $D$ be the domain in $C^{k+1}$ defined by

$$
D=\left\{\left(s_{0}, \cdots, s_{k}\right) \in C^{k+1} ; \operatorname{Re}\left(s_{0}\right)>0, \cdots, \operatorname{Re}\left(s_{k}\right)>0\right\} .
$$

Then one sees that $s^{*}$ is contained in $D$ when $s$ is contained in $D$. By Lemma 2 (iii), one has, for any $i(1 \leq i \leq \nu)$,

$$
F_{i}\left(P_{0}^{m}(\mathrm{grad}) f, s\right)=\varepsilon_{i}\left(\chi_{0}\right)^{m}(-1)^{n m} F_{i}\left(f, s-m \delta\left(\chi_{0}\right)\right)
$$

and

$$
F_{i}^{*}\left(P_{0}^{m}(\operatorname{grad}) f, s\right)=\varepsilon_{i}\left(\chi_{0}\right)^{m}(-1)^{n m} F_{i}^{*}\left(f, s-m \delta^{*}\left(\chi_{0}\right)\right)
$$

if $\operatorname{Re}\left(s_{0}\right), \cdots, \operatorname{Re}\left(s_{k}\right)$ are sufficiently large. Hence we can continue analytically $F_{i}(f, s)$ and $F_{i}^{*}(f, s)$ to holomorphic functions on $D$. Again, by Lemma 2 (iii), one can easily show that the mapping $f \rightarrow F_{i}(f, s)$ (resp. 
$\left.F_{i}^{*}(f, s)\right)$ defines a tempered distribution on the vector space $M(n, R)$ when $s$ is contained in $D$ (cf. Proposition 1.3 in [3]). We call this tempered distribution a zeta distribution associated with the prehomogeneous vector space $(\tilde{G}, \tilde{\rho}, M(n, C))$.

Putting

$$
\Phi(f, s)={ }^{t}\left(\Phi_{1}(f, s), \cdots, \Phi_{\nu}(f, s)\right)
$$

and

$$
\Phi^{*}(f, s)={ }^{t}\left(\Phi_{1}^{*}(f, s), \cdots, \Phi_{\nu}(f, s)\right),
$$

one has the following proposition.

Proposition 1. The vector valued functions $\Phi(f, s)$ and $\Phi^{*}(f, s)$ satisfy a functional equation of the following form

$$
\Phi\left(\hat{f}, s-n \delta\left(\chi_{0}\right)\right)=\gamma\left(s-\delta\left(\chi_{0}\right)\right) C(s) \Phi^{*}\left(f, s^{*}\right),
$$

where $s$ varies in the domain $D$ and $C(s)$ is $a \nu \times \nu$ matrix whose entries $C_{i j}(s)$ are holomorphic in $D$.

This proposition can be proved by the similar argument to Theorem 1.1 in [3]. For the sake of completement, we shall give a proof.

Proof of Proposition 1. For $f \in S(M(n, R))$, set $g \cdot f(x)=f\left(\tilde{\rho}^{*}(g)^{-1} \cdot x\right)$ $\left(g \in \tilde{G}_{R}^{0}\right)$. Then one has $\widehat{g \cdot f}(x)=\chi_{0}^{-n}(g) \hat{f}\left(g^{-1} x\right)$ and hence it follow that

$$
\begin{aligned}
& F_{i}\left(\widehat{g \cdot f}, s-n \chi_{0}\right)=\left|\chi_{0}(g)\right|^{-n} \mid \chi(g)^{s} F_{i}\left(\hat{f}, s-n \chi_{0}\right), \\
& F_{i}\left(g \cdot f, s^{*}\right)=\left|\chi_{0}(g)\right|^{-n}\left|\chi^{*}(g)\right|^{-s^{*}} F_{i}\left(f, s^{*}\right) .
\end{aligned}
$$

On the other hand, one can easily check that $|\chi(g)|^{s}=\left|\chi^{*}(g)\right|^{-s^{*}}$. Then by a theorem of Bruhat (Theorem 3.1 in [7]), there exist holomorphic functions $C_{i j}(s)(1 \leq i, j \leq \nu)$ such that

$$
F_{i}\left(\hat{f}, s-n \chi_{0}\right)=\gamma^{*}\left(s^{*}\right) \sum_{j=1}^{\nu} C_{i j}(s) F_{j}^{*}\left(f, s^{*}\right)
$$

for all $f \in C_{0}^{\infty}\left(M(n, R)-S_{R}^{*}\right)$. We denote by $T_{s}$ a tempered distribution on $M(n, R)$ defined as

$$
T_{s}(f)=F_{i}\left(\hat{f}, s-n \chi_{0}\right)-\gamma^{*}\left(s^{*}\right) \sum_{j=1}^{\nu} C_{i j}(s) F_{j}^{*}\left(f, s^{*}\right) \quad(s \in D) .
$$

One can find a non-negative integer $M$ such that the order of the tempered distribution $T_{s}$ does not exceed $M$ for all $s$ contained in the set $D_{0}=$ $\left\{s \in D ;-1 \leq \operatorname{Re} s_{0} \leq 0\right\}$. If $\delta^{*}(\chi)_{0}, \cdots, \delta^{*}(\chi)_{k} \geq M$, it follows from Lemma 
1.3 in [3] that $T_{s}\left(P_{\chi}^{*} f\right)=0\left(s \in D,-1 \leq s_{0} \leq 0\right.$ and $f \in C_{0}^{\infty}(M(n, R))$. Take a $\chi \in X_{\rho}(\tilde{G})$ such that $\delta^{*}(\chi)_{0}, \cdots, \delta^{*}(\chi)_{k} \geq 0$. From Lemma 2, it follows that, for every $f \in C_{0}^{\infty}\left(M(n, R)-S_{R}\right)$,

$$
\begin{aligned}
& F_{i}\left(\widehat{P_{x}^{*} \cdot f}, s-n \chi_{0}\right) \\
& \quad=(-2 \pi \sqrt{-1})^{-d^{*}(\chi)} \varepsilon_{i}(\chi) \sum_{j=1}^{\nu} C_{i j}(s-\delta(\chi)) \gamma^{*}\left((s-\delta(\chi))^{*}\right) F_{j}^{*}\left(f^{*},(s-\delta(\chi))^{*}\right)
\end{aligned}
$$

and

$$
F_{i}\left(\widehat{P_{\chi}^{*} \cdot f}, s-n \chi\right)=\sum C_{i j}(s) \varepsilon_{j}^{*}(\chi) \gamma^{*}\left(s^{*}+\delta^{*}(\chi)\right) F_{j}^{*}\left(f^{*}, s^{*}+\delta^{*}(\chi)\right) .
$$

Hence, by using the relation $(s-\delta(\chi))^{*}=s^{*}+\delta^{*}(\chi)$, one obtains

$$
C_{i j}(s)=(-2 \pi \sqrt{-1})^{-a^{*}(\chi)} \varepsilon_{i}(\chi) \varepsilon_{j}^{*}(\chi) C_{i j}(s-\delta(\chi)) .
$$

Therefore, for any $f \in C_{0}^{\infty}(M(n, R))$ and $\delta^{*}(\chi)_{0}, \cdots, \delta^{*}(\chi)_{k} \geq M$, one has

$$
T_{s}\left(P_{\chi}^{*} f\right)=(-2 \pi \sqrt{-1})^{-d(\chi)} \varepsilon_{i}(\chi) T_{s-\delta(\chi)}(f) .
$$

This implies that, for any $s \in D_{0}$,

$$
T_{s-\delta(\chi)}(f)=0 \quad\left(f \in C_{0}^{\infty}(M), \delta^{*}(\chi)_{0}, \cdots, \delta^{*}(\chi)_{k} \geq M\right) .
$$

Since $T_{s}$ is a tempered distribution and $T_{s}(f)$ is a holomorphic function of $s$ in $D$, we can conclude $T_{s}(f)=0 \quad(s \in D, f \in S(M(n, R))$ which proves our proposition.

Remark. It is known that the integrals $\Phi_{1}(f, s), \cdots, \Phi_{\nu}(f, s), \Phi_{1}^{*}(f, s), \cdots$, $\Phi_{\nu}^{*}(f, s)$ have analytic continuation to meromorphic functions of $s$ in $C^{k+1}$ (cf. [3], [5]) and hence Proposition 1 holds in $C^{k+1}$.

We denote by $\varepsilon(s)$ the $\nu$ by $\nu$ matrix whose entries are given by

$$
\varepsilon_{i j}(s)=(2 \pi)^{-d(s)} e\left(\frac{d(s)}{4}\right) \varepsilon_{i}(s) \varepsilon_{j}^{*}\left(s^{*}\right), \quad 1 \leq i, \quad j \leq \nu .
$$

Then it is easy to verify the following relation, for any $\chi \in X_{\rho}(\tilde{G})_{R}$,

$$
\varepsilon_{i j}(s)=(-2 \pi \sqrt{-1})^{-a(\chi)} \varepsilon_{i}(\chi) \varepsilon_{j}^{*}(\chi) \varepsilon_{i j}(s-\delta(\chi)) .
$$

We set $t_{i j}(s)=C_{i j}(s) \varepsilon_{i j}(s)^{-1}(1 \leq i, j \leq \nu)$. Then one sees

$$
t_{i \jmath}(s)=t_{i j}(s-\delta(\chi)) \quad\left(\chi \in X_{\rho}\left(\tilde{G}_{R}\right)\right) .
$$

2.2. In this paragraph, we shall give an explicit expression for the functions $t_{i j}(s)$. We denote by $D$ the group consisting of $n$ by $n$ diagonal matrices whose diagonal entries are 1 or -1 . For two subsets $A$ and $B$ 
of $G L(n, R)$, we write $A_{\widetilde{D}} B$ if there exists a matrix $g$ in $D$ such that $A=$ $B \cdot g$. We set, for any integer $i(1 \leq i \leq \nu)$,

$$
K_{i}=\left\{k \in S O(n, R) ;{ }^{\exists} a \in B_{n}^{0}(R) \text { such that } k \cdot a \in V_{i} e_{i}\right\}
$$

and

$$
K_{i}^{*}=\left\{k \in S O(n, R) ;{ }^{\exists} a \in B_{n}^{0}(R) \text { such that } k \cdot a^{*} \in V_{i}^{*} e_{i}\right\},
$$

where

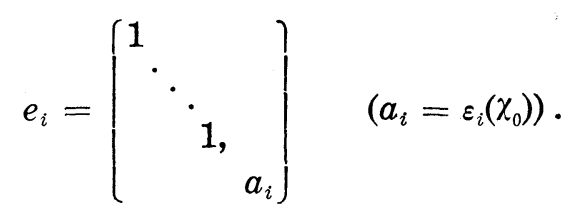

From (A.3), it follows that $V_{i \widetilde{D}} V_{j}(1 \leq i, j \leq \nu)$. On the other hand, the Iwasawa decomposition for the group $G L(n, R)^{0}$ shows that, for any $i(1 \leq i \leq \nu)$,

$$
\begin{aligned}
V_{i} & =K_{i} \cdot B_{n}(R)^{0} \cdot e_{i}, \\
V_{i}^{*} & =K_{i}^{*} \cdot B_{n}(R)_{-}^{0} \cdot e_{i},
\end{aligned}
$$

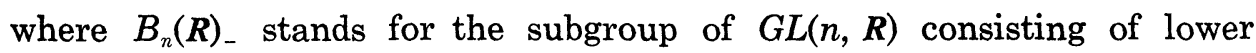
triangular matrices. Since the mapping $x \rightarrow x^{*}$ gives a one-to-one correspondence between $V_{i}$ and $V_{i}^{*}$, one has $K_{i}=K_{i}^{*}(1 \leq i \leq \nu)$.

Using the Iwasawa decomposition

$$
\left(x_{i j}\right)=g \cdot\left(t_{i j}\right), \quad\left(\left(x_{i j}\right) \in G L(n, R)^{0},\left(t_{i j}\right) \in B_{n}(R)^{0}, g \in S O(n, R)\right),
$$

we normalize a Haar measure $d g$ on $S O(n, R)$ by setting

$$
\prod_{1 \leq i, j \leq n} d x_{i j}=\prod_{i=1}^{n} t_{i j}^{n-i} \prod_{i \leq j} d t_{i j} \cdot d g
$$

Then, one has

$$
\int_{K_{i}}|P(g)|^{s} d g=\int_{K_{i}^{*}}\left|P^{*}(g)\right|^{s} d g, \quad(1 \leq i, j \leq \nu) .
$$

Let $f(s)$ be a function on $C^{k+1}$. For a character $\chi \in X_{\rho}(\tilde{G})$, we set:

$$
\sigma_{i}(\chi) f(s)=\varepsilon_{i}(\chi) f(s+\delta(\chi)), \quad(1 \leq i \leq \nu) .
$$

We also set

$$
E_{i}(s)=(-2)^{n}(2 \pi)^{n(n-1) / 2} e\left(-\frac{d(s)}{4}\right) \varepsilon_{i}(-s) \prod_{\ell=1}^{n} \sin \frac{\pi}{2}\left(\lambda_{\ell}(s)-\ell\right) .
$$


We set

$$
\varepsilon_{j}^{*}=\left(\varepsilon_{j}\left(x_{0}^{*}\right), \cdots, \varepsilon_{j}\left(\chi_{r}^{*}\right)\right), \quad 1 \leq j \leq n .
$$

Proposition 2. Assume that, for all $p, q(p \neq q), \varepsilon_{p}^{*} \neq \varepsilon_{q}^{*}$. Then $t_{i j}(s)$ is given by

$$
t_{i j}(s)=\left(\frac{1}{2}\right)^{k+1} \varepsilon_{j}^{*}\left(-s^{*}\right) \prod_{\ell=0}^{k}\left(1+\sigma_{j}\left(\chi_{\ell}\right) E_{i}(s)\right) .
$$

Proof. Let $f$ be a function on the vector space $M(n, R)$ defined by $f(x)=\exp (-\pi(x, x))$. Then $f=\hat{f}$. We make change of variables (4) and (5). Then, using an well known formula:

$$
\frac{1}{2} \pi^{-(1+z) / 2} \Gamma\left(\frac{1+z}{2}\right)=\int_{0}^{\infty} t^{z} e^{-\pi t^{2}} d t
$$

one has

$$
\begin{aligned}
\Phi_{i}(f, s) & =\int_{K_{i}}|P(g)|^{s} d g \cdot \int_{0<t_{\ell}<\infty} \exp \left(-\pi \sum_{\ell=1}^{n} t_{\ell}^{2}\right) \prod_{\ell=1}^{n} t_{\ell}^{\lambda_{\ell}(s)+n-\ell} \prod_{\ell=1}^{n} d t_{\ell} \\
& =\int_{K_{i}}|P(g)|^{s} d g \cdot\left(\frac{1}{2}\right)^{n} \pi^{-\frac{1}{4} n(n+1)} \pi^{-\frac{1}{2} d(s)} \sum_{\ell=1}^{n} \Gamma\left(\frac{\lambda_{\ell}(s)+n-\ell+1}{2}\right)
\end{aligned}
$$

and

$$
\begin{aligned}
\Phi_{i}^{*}\left(f, s^{*}\right) & =\int_{K_{i}^{*}}\left|P^{*}(g)\right|^{*} \cdot \int_{0<t_{\ell}<\infty} \exp \left(-\pi \sum_{\ell=1}^{n} t_{\ell}^{2}\right) \prod_{\ell=1}^{n} t_{\ell}^{* *\left(s^{*}\right)+\ell-1} \prod_{\ell=1}^{n} d t_{\ell} \\
& =\int_{K_{i}^{*}}|P(g)|^{s} d g \cdot\left(\frac{1}{2}\right)^{n} \pi^{-\frac{1}{4} n(n+1)} \pi^{\frac{1}{2} d(s)} \prod_{\ell=1}^{n} \Gamma\left(\frac{-\lambda_{\ell}(s)+\ell}{2}\right) .
\end{aligned}
$$

Thus, from Proposition 1, one obtains

$$
\begin{aligned}
& \frac{1}{\gamma\left(s-n \chi_{0}\right)} \prod_{\ell=1}^{n} \Gamma\left(\frac{\gamma_{\ell}(s)-\ell+1}{2}\right) \\
& \quad=\sum_{j=1}^{\nu} \varepsilon_{i j}(s) t_{i j}(s) \prod_{\ell=1}^{n}\left(\frac{-\lambda_{\ell}(s)+\ell}{2}\right), \quad(1 \leq i \leq \nu) .
\end{aligned}
$$

Using well known formulas of $\Gamma$-function:

$$
\Gamma(z) \Gamma(1-z)=\frac{\pi}{\sin \pi z}
$$

and

$$
\Gamma(2 z)=\frac{2^{z-1}}{\sqrt{ } \pi} \Gamma(z) \Gamma\left(z+\frac{1}{2}\right),
$$

we can rewrite (7) as 
(8) $\sum_{j=1}^{\nu} \varepsilon_{i j}(s) t_{i j}(s)=(-2)^{n}(2 \pi)^{-d(s)+\frac{1}{2} n(n-1)} \prod_{\ell=1}^{n} \sin \pi\left(\frac{\lambda_{\ell}(s)-\ell}{2}\right), \quad(1 \leq i \leq \nu)$.

Hence one has

$$
E_{i}(s)=\sum_{\ell=1}^{\nu} \varepsilon_{\ell}^{*}\left(s^{*}\right) t_{i \ell}(s)
$$

From (6), it follows that

$$
\begin{aligned}
\sigma_{j}(\chi) E_{i}(s) & =\varepsilon_{j}^{*}(\chi) \sum_{\ell=1}^{\nu} \varepsilon_{\ell}^{*}\left((s+\delta(\chi))^{*}\right) t_{i \ell}(s) \\
& =\sum_{\ell=1}^{\nu} \varepsilon_{j}^{*}(\chi) \varepsilon_{\ell}^{*}(\chi) \varepsilon_{\ell}^{*}\left(s^{*}\right) t_{i \ell}(s) .
\end{aligned}
$$

Putting $L_{j}(\chi)=\left\{\ell ; \varepsilon_{j}^{*}(\chi)=\varepsilon_{\ell}^{*}(\chi)\right.$. $\}$, one can rewrite (9) as

$$
\frac{1}{2}\left(1+\sigma_{j}(\chi)\right) E_{i}(s)=\sum_{\ell \in L_{j}(\chi)} \varepsilon_{\ell}^{*}\left(s^{*}\right) t_{i \ell}(s)
$$

Then, by our assumption of Proposition 2, one obtains

$$
\left(\frac{1}{2}\right)^{k+1} \sum_{\ell=0}^{k}\left(1+\sigma_{j}\left(\chi_{\ell}\right)\right) E_{i}(s)=\varepsilon_{j}^{*}\left(s^{*}\right) t_{i j}(s),
$$

which proves our assertion.

By Proposition 1 and Proposition 2, we have the following theorem.

Theorem 1. Assume that, for all $p, q(p \neq q, 1 \leq p, q \leq \nu), \varepsilon_{p}^{*} \neq \varepsilon_{q}^{*}$. Then the tempered distributions $\Phi_{1}(f, s), \cdots, \Phi_{\nu}(f, s), \Phi_{1}^{*}(f, s), \cdots, \Phi_{\nu}^{*}(f, s)$ defined by (2) and (3) satisfy a system of functional equations of the following form.

$$
\left.\Phi\left(\hat{f}, s-n \delta\left(\chi_{0}\right)\right)=\gamma\left(s-n \delta\left(\chi_{0}\right)\right) C(s) \Phi^{*}\left(f, s^{*}\right)\right),
$$

where $C(s)$ is the $\nu$ by $\nu$ matrix whose entries are given by

$$
\begin{gathered}
C_{i j}(s)=(-2)^{n}\left(\frac{1}{2}\right)^{k+1}(2 \pi)^{n(n-1) / 2-d(s)} e\left(\frac{d(s)}{4}\right) \varepsilon_{i}(s) \\
\times \prod_{r=0}^{k}\left(1+\sigma_{j}\left(\chi_{r}\right)\right) e\left(-\frac{d(s)}{4}\right) \varepsilon_{i}(-s) \prod_{\ell=1}^{n} \sin \frac{\pi}{2}\left(\lambda_{\ell}(s)-\ell\right), \\
(1 \leq i, j \leq n) .
\end{gathered}
$$




\section{§3. Examples}

Let $G$ be a connected semi-simple linear algebraic group and $\rho$ an $n$ dimensional irreducible representation both defined over $C$. Assume that the triple $(\tilde{G}, \tilde{\rho}, M(n, C))$ is a prehomogeneous vector space. Then the group $G$ must be one of the following subgroups of $S L(n, C)$ and $\rho$ the identity representation of $G$;

$$
G=S L(n, C), \quad S O(n, C) \text { or } S p(m, C) \text { witn } n=2 m, \quad \text { (cf. [6]) }
$$

Case 1. (cf. [2], [6]) $G=S L(n, C)$.

In this case, $\{\operatorname{det} x\}$ is a complete set of irreducible relative invariants and the $b$-function is given by

$$
\gamma(s)=\Gamma(s+n) \cdots \Gamma(s+1), \quad s \in C .
$$

Since the singular set is given by

$$
S=S^{*}=\{x \in M(n, C), \operatorname{det} x=0\} .
$$

We have the orbit decomposition

$$
M(n, R)-S_{R}=V_{1} \cup V_{2},
$$

where

$$
V_{1}=\{x \in M(n, R), \operatorname{det} x>0\} \quad \text { and } \quad V_{2}=\{x \in M(n, R), \operatorname{det} x<0\} .
$$

Then one has

$$
\begin{aligned}
C_{i j}(s)= & 2^{n-1}(2 \pi)^{n(n-1) / 2-n s} \\
\cdot & \left\{\prod_{\ell=1}^{n} \cos \frac{\pi}{2}(s-\ell+1)+(\sqrt{-1})^{n}(-1)^{i+j} \prod_{\ell=1}^{n} \sin \frac{\pi}{2}(s-\ell+1)\right\}, \\
(1 \leq i, j \leq 2) . &
\end{aligned}
$$

By Theorem 1, one has a system of functional equations.

Propotison 3. The zeta distributions for $G=S L(n, C)$ have the following system of functional equations:

$$
\Phi_{i}(\hat{f}, s-n)=\Gamma(s) \Gamma(s-1) \cdots \Gamma(s-n+1) \sum_{j=1}^{2} C_{i j}(s) \Phi_{j}(f,-s),
$$

where $C_{i j}(s)$ is given by $(11),(i=1,2)$.

Case 2. $G=S O(n, C)$.

For $x \in M(n, R)$, we denote by $x^{i}$ the $i$-th column vector of $x$. Put 


$$
P_{0}(x)=\operatorname{det} x
$$

and

$$
P_{i}(x)=\operatorname{det}\left[\begin{array}{cc}
\left(x^{1}, x^{1}\right), \cdots,\left(x^{1}, x^{i}\right) \\
\vdots & \vdots \\
\left(x^{i}, x^{1}\right), \cdots,\left(x^{i}, x^{i}\right)
\end{array}\right] \quad(1 \leq i \leq n-1)
$$

where $\left(x^{j}, x^{k}\right)$ denotes the usual inner product,

$$
\text { (i.e. } \left.\left(x^{j}, x^{k}\right)=\sum_{\alpha=1}^{n} x_{\alpha}^{j} x_{\alpha}^{k}\right) \text {. }
$$

Then $\left\{P_{0}, \cdots, P_{n-1}\right\}$ is a complete set of irreducible relative invariants of this prehomogeneous vector space, and the singular set $S$ is given by

$$
\begin{aligned}
& S=\cup S_{i}, \\
& S_{i}=\left\{x \in M(n, R) ; P_{i}(x)=0\right\} \quad(0 \leq i \leq n-1) .
\end{aligned}
$$

The orbit decomposition of $M(n, R)-S_{R}$ is given by

$$
M(n, R)-S_{R}=V_{1} \cup V_{2},
$$

where

$$
V_{1}=\left\{x \in M(n, R)-S_{R} ; \operatorname{det} x>0\right\}
$$

and

$$
V_{2}=\left\{x \in M(n, R)-S_{R} ; \operatorname{det} x<0\right\} .
$$

For $s=\left(s_{0}, s_{1}, \cdots, s_{n-1}\right) \in C^{n}$, one sees

$$
\begin{aligned}
& d(s)=n s_{0}+\sum_{\ell=1}^{n-1} 2 \ell s_{\ell} \\
& \lambda_{\ell}(s)=s_{0}+\sum_{m=\ell}^{n} 2 m s_{m}, \quad(1 \leq \ell \leq n),
\end{aligned}
$$

and

$$
\varepsilon_{i}(s)=e\left(\frac{1-(-1)^{i-1}}{4} s_{0}\right), \quad(i=1,2)
$$

Thus one has:

$$
\begin{aligned}
C_{i j}(s)= & 2^{n-1}(2 \pi)^{n(n-1) / 2}\left(\prod_{\ell=1}^{n} \cos \frac{\pi}{2}\left(\lambda_{\ell}(s)-\ell+1\right)\right. \\
& \left.+(-1)^{i+j}(\sqrt{-1})^{n} \prod_{\ell=1}^{n} \sin \frac{\pi}{2}\left(\lambda_{\ell}(s)-\ell+1\right)\right), \quad(1 \leq i, j \leq 2) .
\end{aligned}
$$

From Theorem 1, we obtain the following proposition. 
Proposition 4. The zeta distributions for $G=S O(n, C)$ have the following system of functional equation: for $i=1,2$,

$$
\Phi_{i}\left(\hat{f}, s-n \delta\left(\chi_{0}\right)\right)=\prod_{\ell=1}^{n} \Gamma\left(s_{0}+\sum_{m=\ell}^{n} 2 m s_{m}-\ell+1\right) \sum_{j=1}^{2} C_{i_{\jmath}}(s) \Phi_{\jmath}\left(f, s^{*}\right),
$$

where $C_{i j}(s)$ is given by (12) and

$$
s=\left(s_{0}, s_{1}, \cdots, s_{n-1}\right), \quad s^{*}=\left(-s_{0}-\sum_{m=1}^{n} 2 m s_{m}, s_{2}, \cdots, s_{n-1}\right) .
$$

Case 3. $G=S p(m, C),(n=2 m)$.

Denoting by $[x, y]$ the skew symmetric bilinear form on $C^{n} \times C^{n}$ defined as

$$
[x, y]=\sum_{i=1}^{m}\left(x_{i} y_{\imath}^{\prime}-x_{i}^{\prime} y_{i}\right)
$$

with $x={ }^{t}\left(x_{1}, x_{1}^{\prime}, \cdots, x_{m}, x_{m}^{\prime}\right)$ and $y={ }^{t}\left(y_{1}, y_{1}^{\prime}, \cdots, y_{m}, y_{m}^{\prime}\right)$, we set

$$
P_{0}(x)=\operatorname{det} x
$$

and, for $i=1,2, \cdots, m-1$,

$$
P_{i}(x)=\operatorname{Pff}\left[\begin{array}{ccc}
{\left[x^{1}, x^{1}\right],} & \cdots, & {\left[x^{1}, x^{2 i}\right]} \\
\vdots & & \vdots \\
{\left[x^{2 i}, x^{1}\right],} & \cdots, & {\left[x^{2 i}, x^{22}\right]}
\end{array}\right],
$$

where Pff denotes the Pfaffian.

Then $\left\{P_{0}, \cdots, P_{m-1}\right\}$ is a complete set of the irreducible relative invariants of this prehomogeneous vector space, and the orbit decompositions are given as follows:

$$
M(n, R)-S_{R}=\bigcup_{i \in I} V_{i} \text { and } M(n, R)-S_{R}^{*}=\bigcup_{i \in I} V_{\imath}^{*},
$$

where $I$ denotes a set consisting of all $m$-tuples $\left(i_{0}, \cdots, i_{m-1}\right)$ with each $i_{j}$ is equal to 1 or -1 , and $V_{i}$ is described as

$$
V_{i}=\left\{x \in M(n, R)-S_{R} ; \operatorname{sgn} P_{\ell}=i_{\ell}\right\}, \quad(0 \leq \ell \leq m) .
$$

In this case, one has:

$$
\begin{aligned}
& d(s)=2 m s_{0}+\sum_{\ell=1}^{m-1} 2 \ell s_{\ell}, \\
& \lambda_{\ell}(s)=s_{0}+\sum_{2 i \geq \ell} s_{i},
\end{aligned}
$$




$$
\begin{aligned}
& \gamma(s)=\prod_{\ell=1}^{n} \Gamma\left(s_{0}+\sum_{2 i \geq \ell} s_{i}+n-\ell+1\right), \\
& \varepsilon_{i}(s)=e\left(\frac{1}{4} \sum_{\ell=0}^{m-1} s_{\ell}\left(1-i_{\ell}\right)\right) .
\end{aligned}
$$

Thus one has

$$
\begin{aligned}
C_{i j}(s)= & 2^{m}(2 \pi)^{m(2 m-1)-d(s)} e\left(\frac{d(s)}{4}\right) \varepsilon_{i}(s) \prod_{r=0}^{k}\left(1+\sigma_{j}\left(\chi_{r}\right)\right) \\
& \times e\left(-\frac{d(s)}{4}\right) \varepsilon_{i}(-s) \prod_{\ell=1}^{n} \sin \frac{\pi}{2}\left(s_{0}+\sum_{2 i \geq \ell} s_{i}-\ell\right) .
\end{aligned}
$$

From Theorem 1, we obtain the following proposition.

Proposition 5. The zeta distributions for $G=\operatorname{Sp}(m, C)$ have the following system of functional equations,

$$
\Phi_{i}\left(\hat{f}, s-n \delta\left(\chi_{0}\right)\right)=\prod_{\ell=1}^{n}\left(s_{0}+\sum_{2 i \geq \ell} s_{i}+n-\ell+1\right) \cdot \sum_{j \in I} C_{i j}(s) \Phi_{i}\left(f, s^{*}\right) .
$$

Now, we shall give an example such that $G$ is not reductive. Let $G$ be a subgroup of $S L(n, C)$ consisting of all lower triangular matrices whose diagonal entries are all equal to 1 and $\rho$ a representation of $G$ defined by

$$
\rho(g) x=g \cdot x, \quad x \in M(n, C) .
$$

Then the triplet $(\tilde{G}, \tilde{\rho}, M(n, C))$ is a prehomogeneous vector space. For $x=\left(x_{\alpha \beta}\right) \in M(n, C)$, we set

$$
P_{0}(x)=\operatorname{det} x
$$

and

$$
P_{i}(x)=\operatorname{det}\left[\begin{array}{cc}
x_{11}, \cdots, & x_{1 i} \\
\vdots & \vdots \\
x_{i 1}, \cdots, & x_{i i}
\end{array}\right], \quad(1 \leq i \leq n-1)
$$

One sees that $\left\{P_{0}, \cdots, P_{n-1}\right\}$ is a complete set of irreducible relative invariants of this space and the orbit decomposition is given by

$$
M(n, R)-S_{R}=\bigcup_{i \in I} V_{i}
$$

where $I$ denotes the set of all $n$-tuples $\left(i_{0}, \cdots, i_{n-1}\right)$ with $i_{\ell}=1$ or -1 , and

$$
V_{i}=\left\{x \in M(n, R)-S_{R} ; \operatorname{sgn} P_{\ell}=i_{\ell}, 0 \leq \ell \leq n-1\right\} .
$$


In this case, one has

$$
\begin{aligned}
& d(s)=n s_{0}+\sum_{\ell=1}^{n-1} \ell s_{\ell} \\
& \lambda_{\ell}(s)=s_{0}+\sum_{i \geq \ell} s_{i}, \quad(1 \leq \ell \leq n),
\end{aligned}
$$

and

$$
\varepsilon_{i}(s)=e\left(\frac{1}{4} \sum_{\ell=0}^{n-1} s_{\ell}\left(1-i_{\ell}\right)\right), \quad(i \in I)
$$

Thus, one obtains

$$
\begin{aligned}
C_{i j}(s)= & (-1)^{n}(2 \pi)^{n(n-1) / 2-d(s)} e\left(\frac{d(s)}{4}\right) \varepsilon_{\imath}(s) \\
& \times \prod_{r=0}^{k}\left(1+\sigma_{j}\left(\chi_{r}\right)\right) e\left(-\frac{d(s)}{4}\right) \varepsilon_{i}(-s) \prod_{\ell=1}^{n} \sin \frac{\pi}{2}\left(s_{0}+\sum_{i \geq \ell} s_{\imath}-\ell\right) .
\end{aligned}
$$

By Theorem 1, we have the following proposition

Proposition 6. The zeta distributions for this group have the following system of functional equations, for any $i \in I$

$$
\Phi_{i}\left(\hat{f}, s-n \delta\left(\chi_{0}\right)\right)=\prod_{\ell=1}^{n} \Gamma\left(s_{0}+\sum_{i \geq \ell} s_{i}+n-\ell+1\right) \cdot \sum_{j \in I} C_{i j}(s) \phi_{j}\left(f, s^{*}\right),
$$

where $C_{i j}(s)$ is given by (13).

\section{REFERENCES}

[1] M. Sato and T. Kimura, A classification of irreducible prehomogeneous vector spaces and their invariants, Nagoya Math. J., 65 (1977), 1-155.

[2] M. Sato and T. Shintani, On zeta functions associated with prehomogeneous vector spaces, Ann. of Math., 100 (1974), 131-170.

[ 3 ] T. Shintani, On zeta functions associated with the vector space of quadratic forms, J. Fac. Sci., Univ. Tokyo, 22 (1975), 25-65.

[4] F. Sato, Zeta functions in several variables associated with prehomogeneous vector spaces I: Functional equations, Tôhoku Math. J., The second series, 34, no. 3, (1982), 453-483.

[5] I. N. Bernstein and S. I. Gelfand, Meromorphic property of the functions $P^{\lambda}$, Funct. Anal. Appl., 3 (1969), 68-69.

[6] Y. Teranishi, Relative invariants and $b$-functions of prehomogeneous vector spaces $\left(G \times G L\left(d_{1}, \cdots, d_{r}\right), \tilde{\rho}_{1}, M(n, C)\right)$, Nagoya Math. J., 98 (1985), 139-156.

[7] F. Bruhat, Sur les représentations induites des groupes de Lie, Bull. Soc. Math. France, 84 (1956), 97-205.

Department of Mathematics

Faculty of Science

Nagoya University

Chikusan-ku, Nagoya 464

Japan 